EESTI NSV TEADUSTE AKADEEMIA TOIMETISED. XII KOIDE FOOSIKA-MATEMAATIKA- JA TEHNIKATEADUSTE SEERIA. 1963, NR. 3

ИЗВЕСТИЯ АКАДЕМЙИ НАУК ЭСТОНСКОИ ССР. ТОМ ХІІ СЕРИЯ ФИЗИКО-МАТЕМАТИЧЕСКИХ И ТЕХНИЧЕСКИХ НАУК. 1963, № 3

\title{
О НАПРЯЖЕННЫХ СОСТОЯНИЯХ УПРУГОЙ ПЛИТЫ ПРИ РАСПРОСТРАНЕНИИ СИНУСОИДАЛЬНЫХ ВОЛН ИЗГИБА
}

\author{
А. мяннил \\ у. нигул, \\ кандидат технических наук
}

Анализу корней уравнения Лэмба посвящены многочисленные-работы [1, 2, 3]. Однако напряженные состояния, связанные с этими корнями, в литературе почти не освещены:

В предлагаемой статье исследуются перемещения, моменты, поперечные силы и другие величины при первых трех формах распространения синусоидальных волн изгиба. Приводятся диаграммы относительных амплитуд, а также диаграммы, показывающие распределение перемещений по толщине плиты. Численные данные получены при помощи вычислительной машины $\mathrm{M}-3$, с применением наших формул $\left[{ }^{4}\right]$.

Излагаемые результаты могут представлять интерес при решении конкрегных задач на основе трехмерной теории упругости и позволяют более детально оценить точность двумерных теорий, чем сопоставление корней приближенных характеристических уравнений с корнями уравнения Лэмба. Например, оказывается, что теория типа Тимошенко $\left[{ }^{5}\right.$, $]$ описывает более-менее правильно напряженное состояние формы 1 только при достаточно малых частотах, несмотря на то, что диаграммы фазовой и групповой скоростей хорошо аппроксимируются этой теорией при любых частотах. Еще хуже аппроксимируется напряженное состояние формы 2. Следовательно, область применимости теории типа Тимошенко ограничивается не только тем. что она учитывает лишь две формы изгибных колебаний, а также условием малости частот.

\section{1. Обозначения и расчетные формулы}

Рассмотрим напряженные состояния изгибного типа, зависящие от продольной координаты $x$, нормальной координаты $z$ и времени $t$. Введем безразмерные координаты $\xi$, $\zeta$ и безразмерное время $\tau$ по формулам:

$$
\xi=\frac{x}{h}, \quad \zeta=\frac{z}{h}, \quad \tau=\frac{t_{c_{2}}}{h},
$$

где $h$ - полутолщина плиты и $c_{2}-$ скорость распространения волн сдвига.

Предположим, что поверхности плиты $\zeta= \pm 1$ не нагружены, и будем иметь в виду напряженные состояния, зависящие от $\xi$, $\tau$ через функцию

$$
\Phi=A \exp (i \varkappa \xi+i \Omega \tau), \quad A=\text { const, }
$$

где волновое число $\chi$ и безразмерная частота $\Omega$ являются вещественными величинами.

4 ENSV TA Toimetised T-3 1963 
Безразмерную длину волн $l$ и фазовую скорость $\psi$ определим по формулам

$$
l=\frac{\pi}{x}, \quad \psi=\frac{\Omega}{x} .
$$

Примем еще обозначения: $E-$ модуль упругости, $\mu-$ коэффициент Пуассона, $u_{\xi}, u_{\xi}$ - перемещения, $M_{\xi}$ - изгибающий момент, $Q_{\xi}-$ поперечная сила, б६ - напряжение изгиба.

Будем рассматривать величины:

и отношения:

$$
u, u_{1}, U ; w, w_{0}, w_{1}, W ; M, Q ; \sigma, \sigma_{1}
$$

$$
u_{1}^{*}=u_{1} / W, \quad U^{*}=U / W, \quad w_{0}^{*}=w_{0} / W, \ldots
$$

Величины (1.4) имеют следующий смысл:

$$
\begin{aligned}
& u_{\xi}=u i \Phi, \quad u_{1}=u(\zeta=1), \\
& u_{\zeta}=w \Phi, \quad w_{0}=w(\zeta=0), \quad w_{1}=w(\zeta=1), \\
& U=\frac{3}{2} \int_{-1}^{+1} u \zeta d \zeta, W=\frac{1}{2} \int_{-1}^{+1} w d \zeta, \\
& M_{\xi \xi}=\frac{E h^{2}}{1-\mu^{2}} M \Phi, \quad Q_{\xi}=\frac{E h}{1-\mu^{2}} i Q \Phi, \\
& \sigma_{\xi \xi}=\frac{E h^{2}}{1-\mu^{2}} \sigma \Phi, \quad \sigma_{1}=\sigma(\zeta=1) .
\end{aligned}
$$

\section{а. Формуль трехмерной теории} Пэмба

В трехмерной теорий соотношение между $\varkappa$ и $\Omega$ дается уравнением

$$
\gamma^{4} \cos \beta \frac{\sin \alpha}{\alpha}+x^{2} \beta^{2} \cos \alpha \frac{\sin \beta}{\beta}=0
$$

где

$$
\alpha^{2}=\frac{1-2 \mu}{2-2 \mu} \Omega^{2}-x^{2}, \quad \beta^{2}=\Omega^{2}-x^{2}, \quad \gamma^{2}=\frac{1}{2} \Omega^{2}-x^{2} .
$$

Формулы для исследуемых величин (1.4) легко выписать на основе соответствующих выражений работы [4]. В рассматриваемом случае изгибные волны двух видов, указанные в [ $\left.{ }^{4}\right]$, отличаются лишь множителем $B=B(\varkappa, \Omega)$. Имея в виду анализ отношений величин (1.4), достаточно рассмотреть волны одного вида. Итак, воспользуемся формулами работы $\left[{ }^{4}\right]$, полагая, что $\Phi_{1} \equiv \Phi_{2} \equiv 0, \Phi_{3}=\Phi$. Учитывая уравнение $(1.6)$, получим формулы:

$$
\begin{gathered}
u=\frac{2 \varkappa}{\Omega^{2}}\left(\gamma^{2} \cos \beta \frac{\sin \alpha \zeta}{\alpha}-\beta^{2} \cos \alpha \frac{\sin \beta \zeta}{\beta}\right), \\
U=-\frac{3 x}{(1-\mu) \alpha^{2} \gamma^{2}}\left[\left(\frac{1-2 \mu}{2} \Omega^{2}+\mu x^{2}\right) \frac{\sin \beta}{\beta}+\mu \gamma^{2} \cos \beta\right] \cos \alpha \\
w=\frac{2}{\Omega^{2}}\left(\gamma^{2} \cos \beta \cos \alpha \zeta+x^{2} \cos \alpha \cos \beta \zeta\right), \quad W=\frac{\gamma^{2}}{\beta^{2}} \cos \beta \frac{\sin \alpha}{\alpha}
\end{gathered}
$$




$$
\begin{gathered}
M=\frac{\Omega^{2}}{\alpha^{2} \gamma^{2}}\left(x^{2} \frac{\sin \beta}{\beta}+\mu \gamma^{2} \cos \beta\right) \cos \alpha \\
Q=-\frac{(1-\mu) x \Omega^{2}}{\gamma^{2}} \cos \alpha \frac{\sin \beta}{\beta}, \quad \sigma_{1}=\frac{x^{2} \beta^{2}}{\gamma^{2}} \cos \alpha \frac{\sin \beta}{\beta} .
\end{gathered}
$$

\section{6. Формулы теории типа Тимошенко}

$$
\begin{gathered}
\Omega^{2}-\frac{2}{3(1-\mu)}\left(\frac{1-\mu}{2} \Omega^{2}-x^{2}\right)\left(\frac{1}{k^{2}} \Omega^{2}-x^{2}\right)=0 \\
u=-x_{5}, W=1+\frac{2}{3(1-\mu) k^{2}}\left(x^{2}+\frac{1-u}{2} \Omega^{2}\right) \\
M=\frac{2}{3} x^{2}, \quad Q=\frac{2}{3} x\left(x^{2}-\frac{1-\mu}{2} \Omega^{2}\right)
\end{gathered}
$$

где $k^{2}$ обозначает коэффициент сдвига $\left[{ }^{5,6}\right]$.

\section{в. Приближеннпе формуль для случая $x_{m} \ll 1$}

Пусть $x_{m}=\varkappa_{m}(\Omega) \quad(m=1,2,3)$ обозначают первые три корня уравнения Лэмба (1.6), начинающиеся (имеющие нулевые значения) соответственно при

$$
\Omega=0, \quad \Omega=\Omega_{m}^{0}=\frac{n \pi}{2}, \quad(n=2 m-3, m \geqslant 2) .
$$

При $\Omega \rightarrow 0$ напряженное состояние формы 1 приближенно определяется известными формулами теории Кирхгоффа, а при $\Omega \rightarrow \Omega_{m}^{0}$ имеех для формы $m$ следующие приближенные формулы [ $\left.{ }^{4}\right]$ :

$$
l \approx \frac{\pi^{2} R}{\Omega^{2}-\frac{n^{2} \pi^{2}}{4}}, \quad R \approx \frac{32 \alpha}{n^{2} \pi^{2} \operatorname{tg} \alpha}, \quad a \approx \frac{n \pi}{2} \sqrt{\frac{1-2 \mu}{2-2 \mu}} .
$$

На основе (1.12) можно получить из (1.8) приближенные формулы для величин (1.4). Например, для перемещений имеем формулы:

$$
\begin{gathered}
u=\frac{n \Omega^{2} l^{3}}{\pi^{2}} \cos \alpha \sin \beta \zeta, \\
w=\frac{2 \Omega^{2} l^{2}}{\pi^{2}}\left[\cos \alpha \cos \beta \zeta+(-1)^{\frac{n-1}{2}} \frac{n \pi(1-R)}{8}\right] .
\end{gathered}
$$

\section{2. Численные результаты}

При исследовании выражений (1.4) можно выбрать параметром либо $\Omega$, либо $l$, или же какую-нибудь другую величину, связанную с ними. При выборе параметров было учтено, что при частотах, близких к $\Omega_{m}^{0}$, величины (1.4) являются быстроизменяюшимися функциями от $\Omega$ и медленноизменяющимися функциями от $l$, а при других значениях $\Omega$ наоборот. Поэтому составлены диаграммы двух видов. На рис. 1, 2, 4, $5,7,8$ исследуемые величины изображены как функции от $\Omega$, а на рис. 3 , 6 - как функции от $l$. При этом рис. 3,6 охватывают те диапазоны, при которых величины (1.4) являются быстроизменяющимися функциями от частоты $\Omega$. 

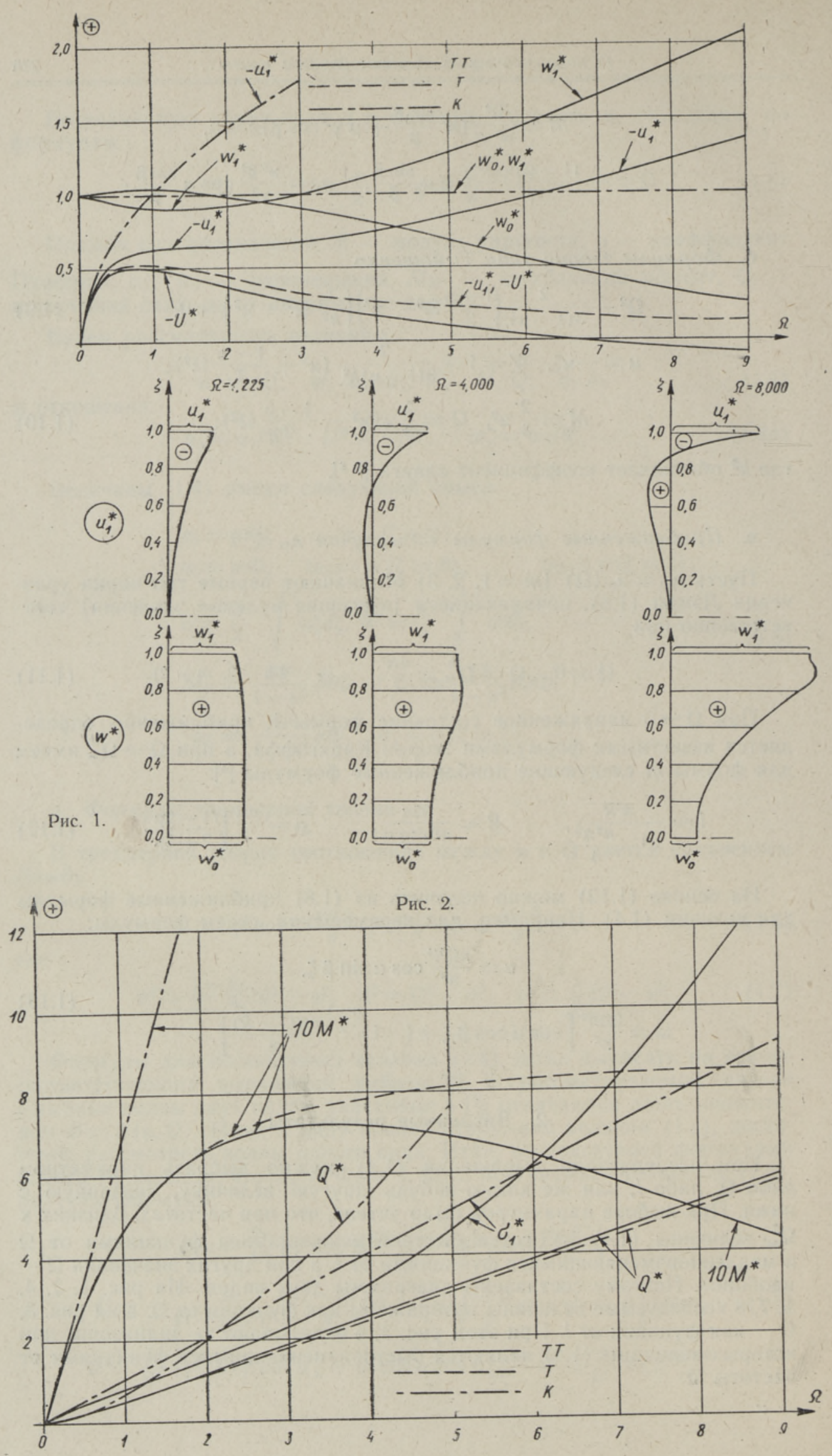
Во всех расчетах был принят коэффициент Пуассона $\mu=0,3$ и в теории типа Тимошенко коэффициент сдвига $k^{2}=0,860$. В расчетах, связанных с составлением рис. $1,2,4,5,7,8$, были применены численные значения корней $\chi_{m}=\chi_{m}(\Omega)$ из [3], а в расчетах, связанных с составлением рис. 3,6 - данные из табл. 1, вычисленной на основе (1.6) по заданным значениям $l$ при помощи итерационного процесса. Қак при составлении табл. 1, так и при вычислении величин (1.4), была применена вычислительная машина M-3.

Рис. 1,2 посвящены форме 1 , рис. $3,4,5$ - форме 2 , рис. $6,7,8-$ форме 3. Изложенные диаграммы іозволяют определить отношения амплитуд величин (1.4) и дают информацию о распределении перемещений по толщине плиты. (Диаграммы распределения перемещений даны на рис. $1,3,6$ для верхней полутолщины плиты).

Рис. 1 наглядно показывает, как на форме 1 с увеличением $\Omega$ почтн линейное распределение перемещений постепенно переходит в распределение, типичное для поверхностных волн Релея.

Для форм 2, 3 распределение перемещений по толщине плиты (рис. 3,6 ) хорошо аппроксимируется при достаточно длинных волнах $l$ формулами (1.13). Это связано с тем, что распределение тангенциальных перемещений $u$ форм 2,3 имеет при $l \geqslant 2$ явно выраженный синусоидальный характер $(1 / 2$ и $3 / 2$ волн соответственно). В случае формы 3 нормальные перемещения ш также распределяются при $l \geqslant 2$ по закону, близкому к синусоидальному.

\section{3. О точности и применимости двумерных теорий}

Двумерные теории обыкновенно строятся на основе предположений

$$
\text { а) } w \approx \text { const, б) } u \approx \zeta \text { const или } \sigma \approx \zeta \text { const. }
$$

При достаточно малых значениях $\Omega$ эти предположения приближенно выполняются на форме 1 (рис. 1) и более грубо - также на форме 2 (рис. 3). Указанное согласуется с тем, что при аналитическом выводе из (1.6), (1.8) формул двумерных теорий приходится воспользоваться $\left.{ }^{4}\right]$ предположениями о малости величин типа $\Omega$ и ж.

Рис. 1. Форма 1. ТТ - трехмерная теория, Т - теория типа Тимошенко, $\mathrm{K}-$ теория Кирхгоффа (прямая $w_{0}^{*}=w_{1}^{*}=1$ соответствует теориям Кирхгоффа н типа Тимошенко).

Рнс. 2. Форма 1. ТТ - трехмерная теория, Т - теория типа Тимошенко, К теория Кирхгоффа. 


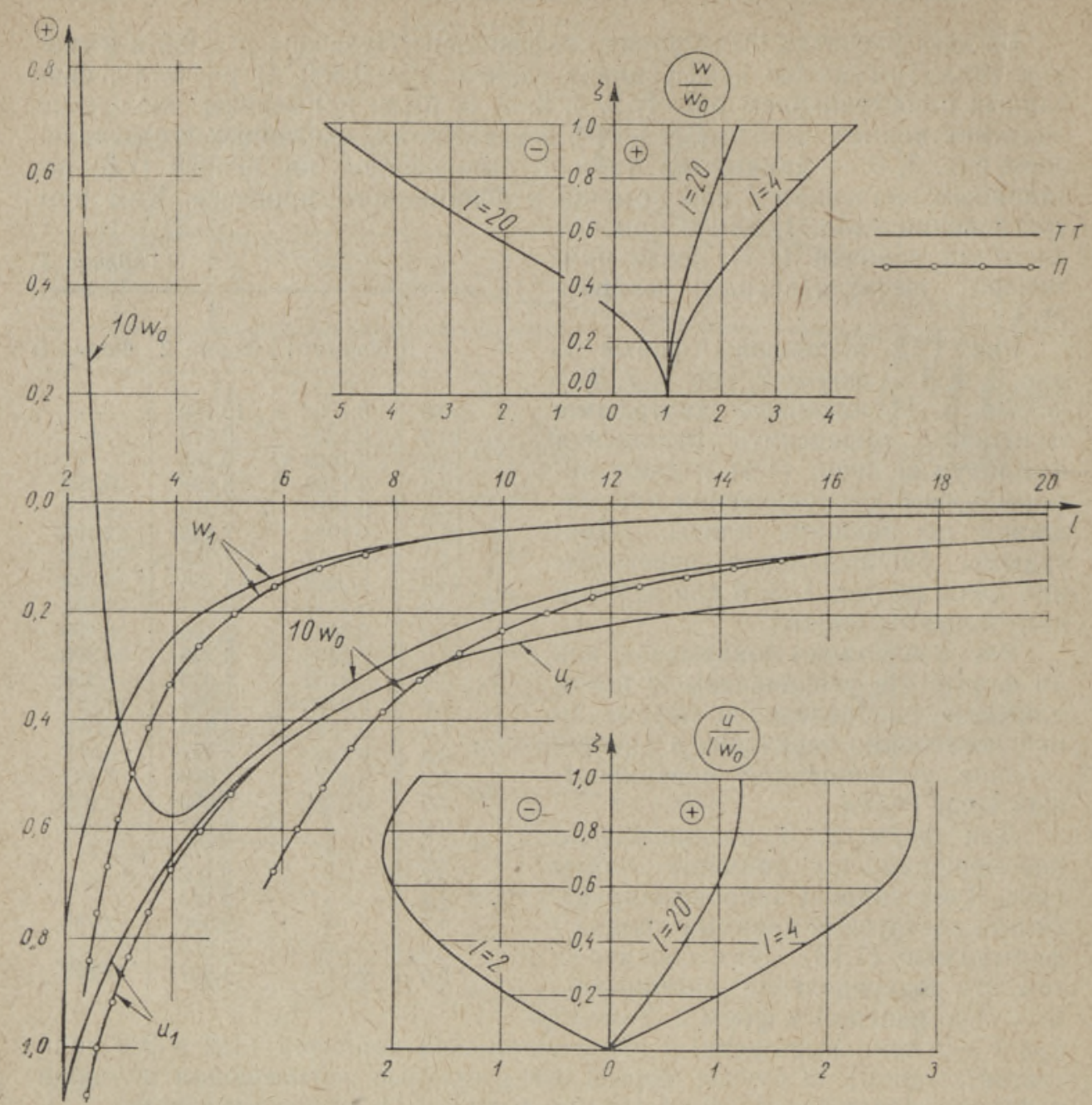

Рис. 3. Форма 2. ТТ - трехмерная теория, П - приближенные формулы (1.13).

В количественном смысле область применимости гипотез двумерных теорий является при той же погрешности различной. В качестве примера в табл. 2 приведены некоторые данные для формы 1.

Из табл. 2 видно, что область применимости гипотез 1а, 16 шире областей применимости остальных гипотез. Гипотеза 3 , применяемая В теории Қирхгоффа, имеет гораздо более ограниченную область применимости, чем гипотезы 2a, 2б, заменяющие ее в уточненных теориях. Поэтому область применимости уточненных теорий шире, чем теории Кирхгоффа. Кроме того, уточненные теории аппроксимируют еще форму 2. Хотя гипотезы типа (3.1) являются в случае формы 2 сравнительно грубыми, они все-же позволяют приближенно определить некоторые интегральные величины.

О точности теории Кирхгоффа можно судить по диаграммам на рис. 1,2 . Видно, что при $\Omega \sim 1$ погрешность является уже весьма большой.

Остановимся еще на точности теории типа Тимошенко при определении отношений между интегральными величинами. Такие отношения 


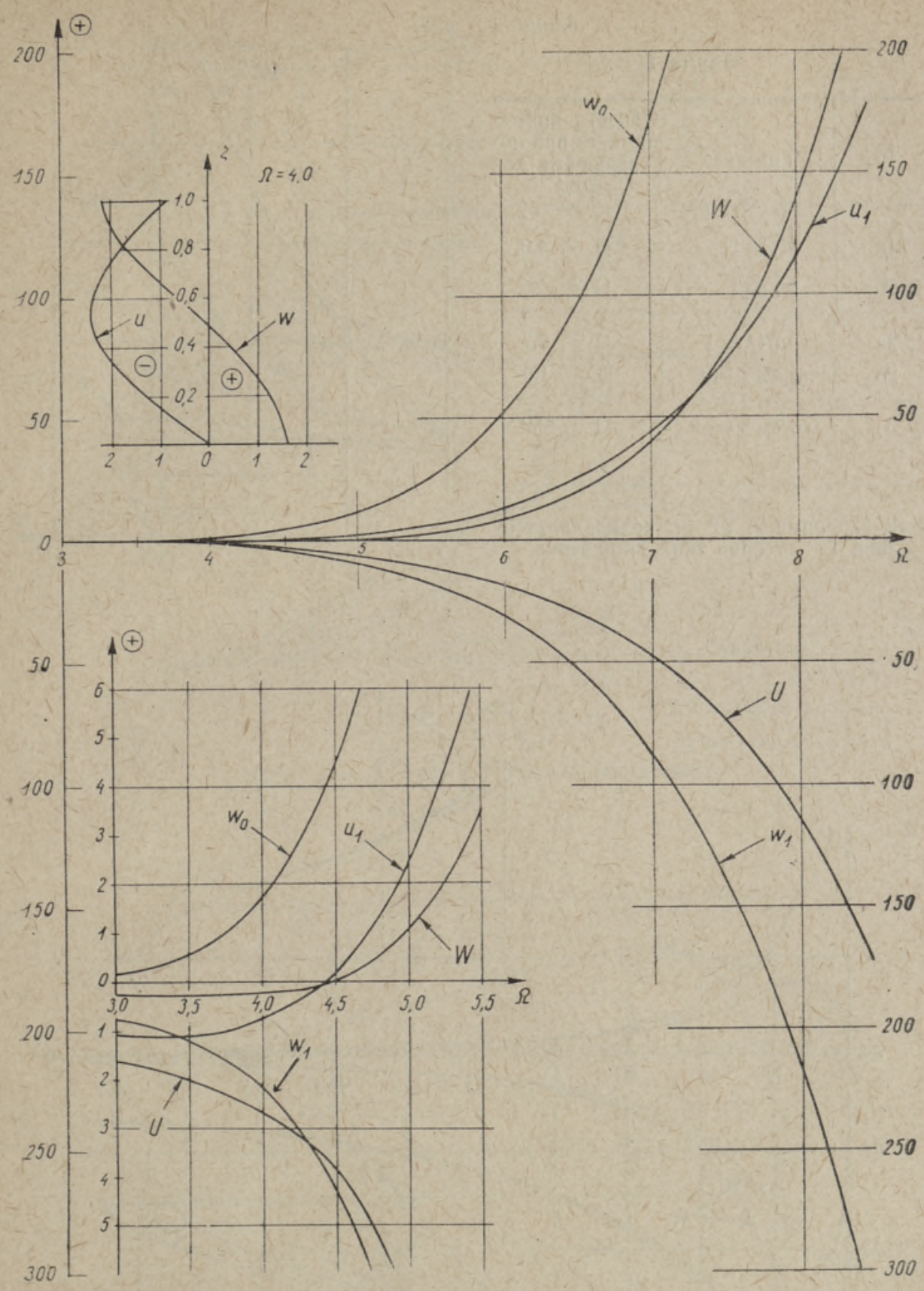

Рис. 4. Форма 2. Трехмерная теория.

$U^{*}, M^{*}, Q^{*}$ показаны на рис. $1,2,5$. В пределах погрешности $20 \% U^{*}$ определяется для формы 1 при $\Omega \leqslant 2, M^{*}-$ при $\Omega \leqslant 7$ и $Q^{*}$ - до весьма большой частоты. При форме 2 погрешности вычисления $U^{*}$, $M^{*}, Q^{*}$ возрастают более одинаково и превышают $20 \%$ при $\Omega \sim 3$. Здесь не учтена погрешность, связанная с тем, что кривая $x_{2}=x_{2}(\Omega)$. 

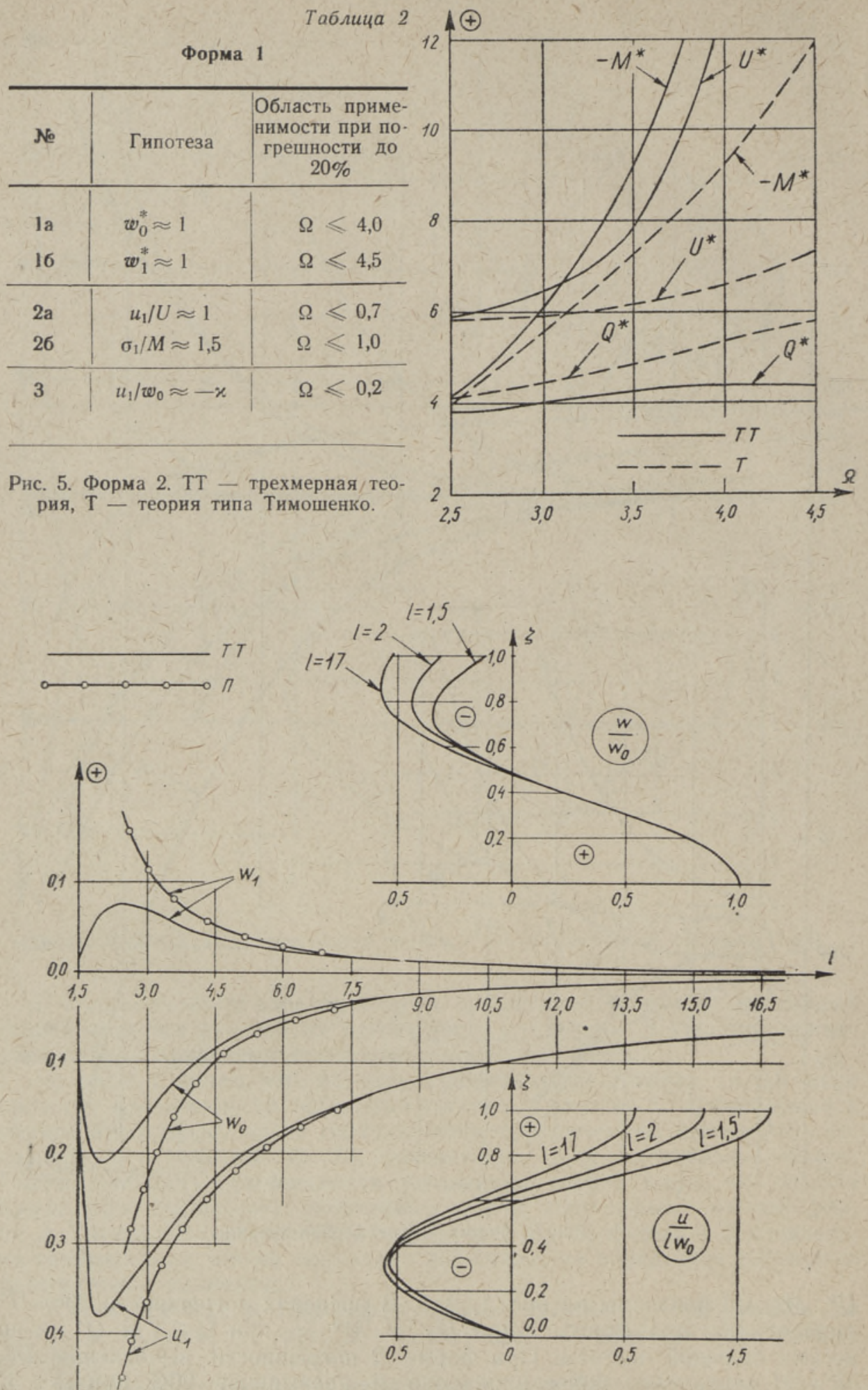

Рис. 6. Форма 3. ТТ - трехмерная теория, П - приближенные формулы (1.13). 


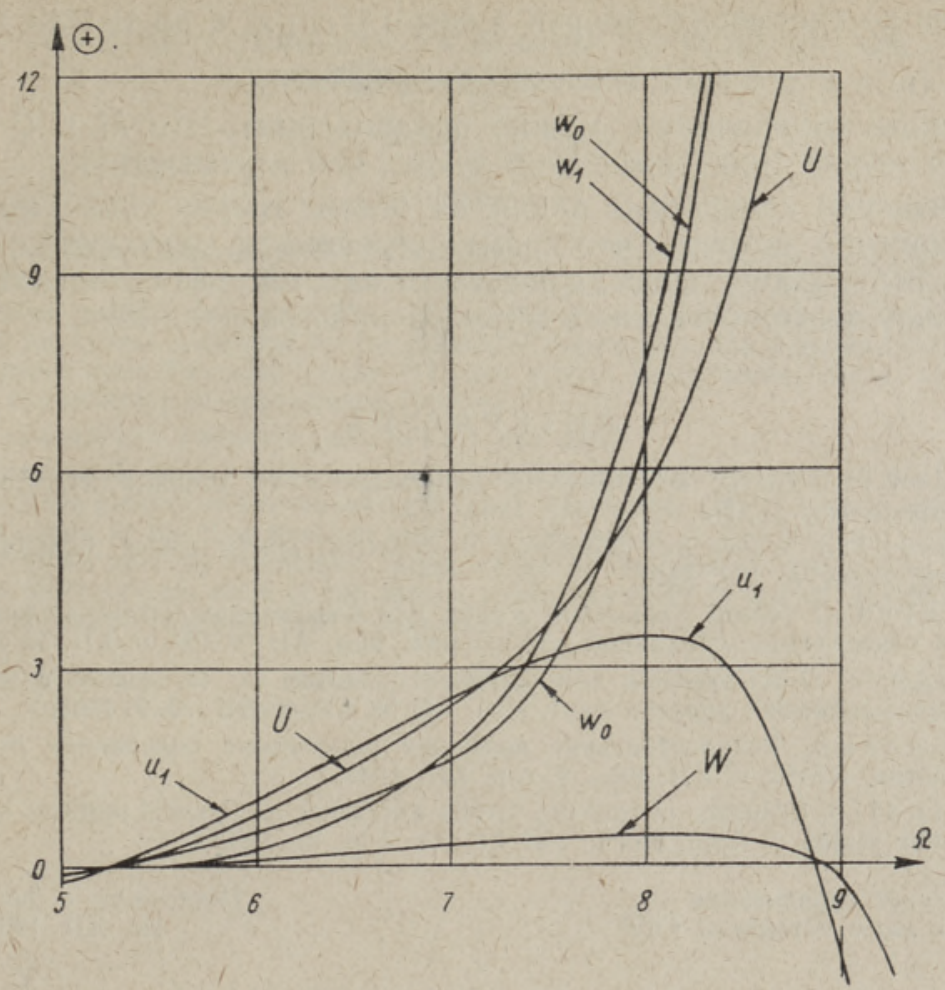

Рнс. 7. Форма 3. Трехмерная теорня.

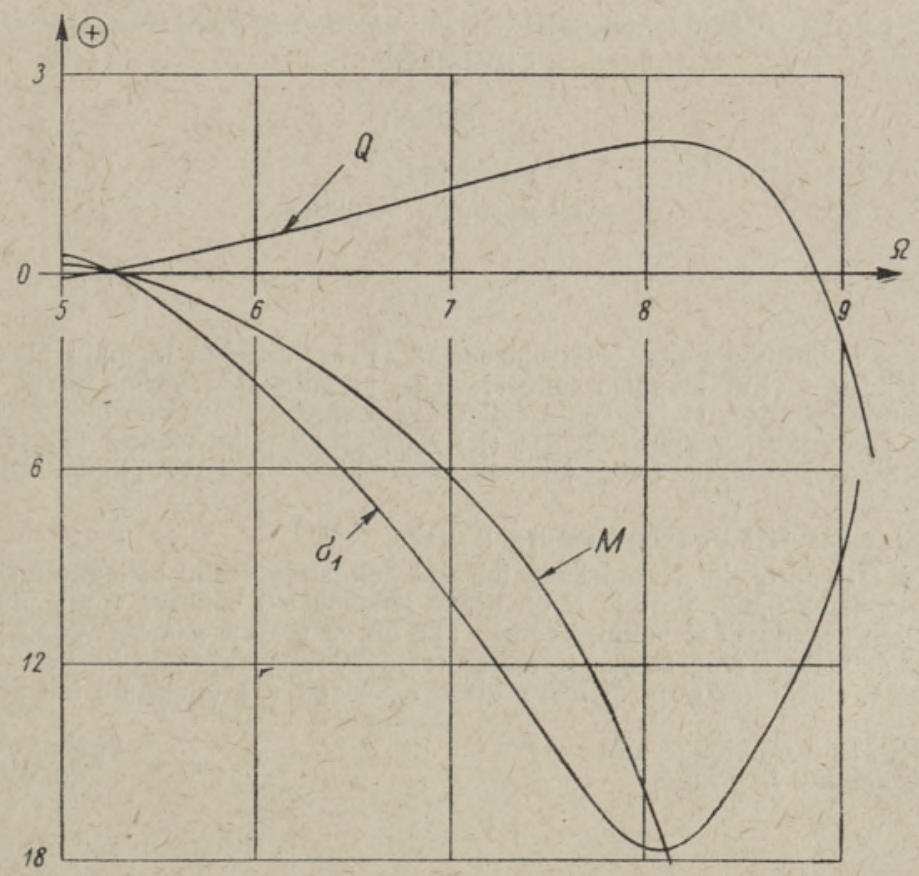

Рнс. 8. Форма 3. Трехмерная теория. 
начинается по трехмерной теории в точке $\Omega=\frac{1}{2} \pi$, а по теории типа Тнмошенко (при $\kappa^{2}=0,860$ ) в точке $\Omega=\sqrt{3 \kappa^{2}}=1,61$.

На основе изложенного можно предположить, что $M$ и $Q$ определяются по теории типа Тимошенко лучше, чем перемещения.

При решении конкретных задач, на основе теории типа Тимошенко, следует, конечно, помнить, что кроме погрешности, связанной с аппроксимацией первых двух форм, существует еще погрешность, связанная с отбрасыванием всех остальных форм распространения волн.

\title{
ЛИТЕРАТУРА
}

1. J. Miklowitz, Recent developments in elastic wave propagation, Appl. Mech. Reviews, Vol. 13, No. 12, p. 865, 1960.

2. R. D. Mindlin, Waves and vibrations in isotropic, elastic plates, Structural Mech., Perg. Press, p. 199, 1960.

3. У. К. Н и г ул, О корнях уравнения Лэмба для деформации плиты, антисимметричной относительно срединной поверхности, Изв. АН ЭССР, т. ХІІ, № 3, 1963.

4. У. К. Ни гул, О примененни символического метода А. И. Лурье в трехмерной теории динамики упругих плит, Изв. АН ЭССР, т. ХII, № 2, 1963.

5. Я. С. Уфлянд, Расиространение волн при поперечных колебаниях стержней и пластин, ПММ, т. XII, вып. 3, стр. 287, 1948.

6. R. D. Mind $1 \mathrm{i}$, Influence of rotatory inertia and shear on flexural motions of isotropic elastic plates, J. Appl. Mech., Vol. 18, No. 1. p. 31, 1951.

\author{
Институт кибернетики \\ Академии наук Эстонской ССР
}

Поступила в редакцию

25. XII 1962

\section{ELASTSE PLAADI PINGEOLUKORDADEST SINUSOIDAALSETE PAINDELAINETE LEVIMISEL}

\author{
A. Männil \\ U. Nigul, \\ tehnikateaduste kandidaat
}

\section{Resümee}

Kasutades kolmemõôtmelist elastsusteooriat (TT), uuritakse lõpmatu plaadi pingeolu: kordi paindelainete (1.2) levimise esimese, teise ja kolmanda vormi puhul. Numbrilised tulemused suuruste (1.4), (1.4a) kohta esitatakse joonistel 1-8. Vōrdluseks tuuakse kõve$\mathrm{rad}$, mis on arvutatud Kirchhoffi teooria (K), Timošenko tüüpi teooria $(\mathrm{T})$ ja ligikaudsete valemite (1.13) (П) alusel. Need kõverad võimaldavad hinnata ligikaudsete teooriate täpsust.

Arvutustes kasutati Poisson'i tegurit $\mu=0,3$.

Valemid (П) aproksimeerivad hästi teise ja kolmanda vormi kohamuutusi, kui dimensioonita lainepikkus $l \geqslant 2$. Teooria $K$ on heaks lähenduseks esimese vormi pingeolukorrale väga madalate sageduste $\Omega$ puhul. Teooria (T) on kasutatav esimese vormi puhul tunduvalt laiemas sageduste diapasoonis ja annab teatavat informatsiooni ka teise vormi pingeolukorra kohta. Seejuures $Q$ ja $M$ mäăratakse teooriaga (T) täpsemini kui kohamuutused. Eesti NSV Teaduste Akadeemia
Küberneetika Instituut
Saabus toimetusse 25. XII 1962 


\title{
ON STRESS STATES OF AN ELASTIC PLATE AT MOTION OF BENDING SINE WAVES
}

\author{
A. Männil, U. Nigul
}

\section{Summary}

Using the three-dimensional theory of elasticity (TT) the stress states of an infinite plate are studied at the first, second and third mode of bending waves (1.2). The numerical results for quantities (1.4), (1.4a) are presented on figs. 1-8. For comparison the curves computed by the Kirchhoff theory $(\mathrm{K})$, the Timoshenko type theory (T) and approximate formulae $(1,13)$ (П) are given. These curves could be used for estimating the accuracy of approximate theories.

In all calculations Poisson's ratio $\mu=0.3$ was used.

The formulae $\Pi$ give a good approximation for the displacements of the second and third mode if the dimensionless wavelength $l \geqslant 2$. The theory $(\mathrm{K})$ is a good approximation of the stress state of the first mode at very low frequencies $\Omega$. The theory (T) is appliable by the first mode over an essentially larger range of frequencies, and it also gives some information on the stress state of the second mode. Herewith $Q$ and $M$ are determined by the theory (T) more precisely than displacements.

\author{
Academy of Sciences of the Estonian S.S.R., \\ Institute of Cybernetics
}

Received

Dec. 25th, 1962 